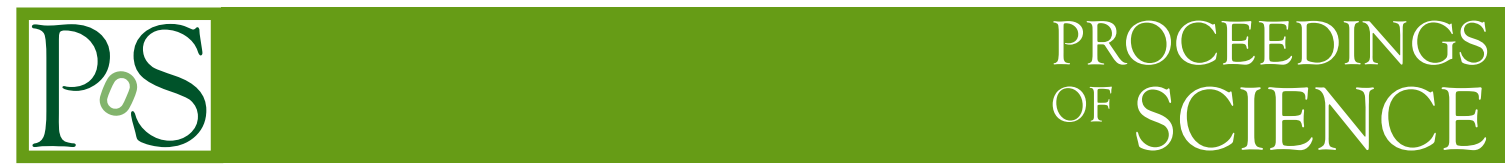

\title{
Charged massive spin two particles
}

\author{
Yuichi Ohara*† \\ Nagoya university \\ E-mail: yuichi@th.phys.nagoya-u.ac.jp
}

We constructed models of self-interacting charged massive spin two particles by using the interaction proposed by Hinterbichler. Then, we investigate the some basic properties of this theory.

The 3rd International Symposium on "Quest for the Origin of Particles and the Universe"

5-7 January 2017

Nagoya University, Japan

* Speaker.

${ }^{\dagger}$ A footnote may follow. 


\section{Introduction}

Theories of massive spin two particles have been intensively studied recently inspired by the success of the construction of the ghost-free massive gravity known as the dRGT theory [1, 2, 3, 4]. Especially, there are lots of works in the application of the theory to the cosmology with the expectation of the (partial) resolution of the cosmological constant problem. While many difficulties for the phenomenological applications have been pointed out alredy, there is room to discuss field theoretical apects of massive spin two particles.

In this study, we consider interacting theories of non-gravitational, charged massive spin two particles. Since the particles are not gravitons anymore, the kinetic term is not necessary to be the fully nonlinear Einstein-Hilbert action. Led by this consideration, we construct two non-gravitational theories by adding interactions discovered by Hinterbichler [5] to the linearized Einstein-Hilbert action. The remarkable property of the interactions is that they are free from the Boulware-Deser like ghost and could have nontrivial vacua. Thus, we discuss the structure of the theory around nontrivial vacua.

\section{Free field theory}

In 1939, the Lagrangian of the free massive spin two particles was prposed by Fierz and Pauli [6]. Although the theory does not have any gauge symmetry, the form of the mass term is uniquely determined by the requirement of the absense of ghosty modes.

$$
\mathscr{L}_{\mathrm{FP}}=-\frac{1}{2} \partial_{\lambda} h_{\mu v} \partial^{\lambda} h^{\mu v}+\partial_{\mu} h_{v \lambda} \partial^{v} h^{\mu \lambda}-\partial_{\mu} h^{\mu v} \partial_{v} h+\frac{1}{2} \partial_{\lambda} h \partial^{\lambda} h-\frac{1}{2} m^{2}\left(h_{\mu v} h^{\mu v}-h^{2}\right)
$$

This Lagrangian is called the FIerz-Pauli Lagrangian. As shown, the relative coefficient between $h_{\mu v} h^{\mu v}$ and $h^{2}$ is tuned to be -1 . If the coefficient changes, the ghost mode appears and theory loses the consistency.

\section{Interactions}

As the massive theory with finte spin can not be renormalizable, we should regard the massive spin two field theory as an effective field theory, which means that any interactions are allowed as far as the global symmetry is respected. However, the some class of interactions changes the property of the theory dramatically. For instance, massive gravity can not be a gravitational theory unless we carefully chose the potential term because the Boulware-Deser ghost [7] breaks the quantum mechanical consistency in curved backgrounds. The essential point in the construction of the dRGT theory is that the fully nonlinear potential terms keep the degree of freedom which the free field theory has. Inspired by this fact, we consider non-gravitational, self-interacting massive spin two particles using the following "ghost-free" interactions for the free Lagrangian.

$$
\mathscr{L}_{3} \sim \eta^{\mu_{1} v_{1} \mu_{2} v_{2} \mu_{3} v_{3}} h_{\mu_{1} v_{1}} h_{\mu_{2} v_{2}} h_{\mu_{3} v_{3}}, \quad \mathscr{L}_{4} \sim \eta^{\mu_{1} v_{1} \mu_{2} v_{2} \mu_{3} v_{3} \mu_{4} v_{4}} h_{\mu_{1} v_{1}} h_{\mu_{2} v_{2}} h_{\mu_{3} v_{3}} h_{\mu_{4} v_{4}} .
$$

Here $\eta^{\mu_{1} v_{1} \cdots \mu_{n} v_{n}}$ is defined as the product of $n \eta_{\mu v}$ given by antisymmetrizing the indices $v_{1}, v_{2}$, $\cdots$, and $v_{n}$. Since we only consider the $Z_{2}$ and $U(1)$ symmetries, the cubic interaction is irrelevant to the following discussion. 


\section{Interacting theory}

Let us move on to writting down the Lagrangian which has $Z_{2}$ or $U(1)$ symmetry. The Lagrangian which is invariant under the $Z_{2}$ transformation $h_{\mu v} \rightarrow-h_{\mu v}$ is given by

$$
\mathscr{L}=\mathscr{L}_{F P}+\frac{\lambda}{4 !} \eta^{\mu_{1} v_{1} \mu_{2} v_{2} \mu_{3} v_{3} \mu_{4} v_{4}} h_{\mu_{1} v_{1}} h_{\mu_{2} v_{2}} h_{\mu_{3} v_{3}} h_{\mu_{4} v_{4}}
$$

while the $U(1)$ invariant theory is

$\mathscr{L}=\eta^{\mu_{1} v_{1} \mu_{2} v_{2} \mu_{3} v_{3}} \partial_{\mu_{1}} h_{\mu_{2} v_{2}}^{\dagger} \partial_{v_{1}} h_{\mu_{3} v_{3}}+m^{2} \eta^{\mu_{1} v_{1} \mu_{2} v_{2}} h_{\mu_{1} v_{1}}^{\dagger} h_{\mu_{2} v_{2}}+\frac{\lambda}{3 !} \eta^{\mu_{1} v_{1} \mu_{2} v_{2} \mu_{3} v_{3} \mu_{4} v_{4}} h_{\mu_{1} v_{1}}^{\dagger} h_{\mu_{2} v_{2}} h_{\mu_{3} v_{3}}^{\dagger} h_{\mu_{4} v_{4}}$

\section{Vacuum structure in the $Z_{2}$ theory}

An ansatz of Lorentz-invariant vacua is given by $h_{\mu \nu}^{\mathrm{VEV}}=C \eta_{\mu \nu}$ where $C$ is consant. Then, the theory has nontrivial extrema under the following parameter region $\llbracket 8]$.

$$
\left\{\begin{array}{l}
\lambda<0 \quad \text { and } \quad \mathrm{m}^{2}>0 \\
\lambda>0 \text { and } \mathrm{m}^{2}<0
\end{array} .\right.
$$

The stability analysis of the vacua is given as follows.

(a) $\lambda<0$ and $m^{2}>0$

The trivial vacuum is only stable. The energy density of the stable vacuum is higher than the two unstable, nontrivial vacua. vacuum.

(b) $\lambda>0$ and $m^{2}<0$

The theory has two stable nontrivial vacua while the trivial vacuum is unstable in the sense that the tachyonic state is absent. It should be noted that the energy density of the stable vacua is higher than that of the trivial vacuum.

We should emphasize here that vacua which have higher energies are stable.

\section{Vacuum structure in the $U(1)$ theory}

From the above observation, we could expect that a Nambu-Goldstone boson appears in a nontrivial vcuum when the parameters set to be $\lambda>0$ and $m^{2}<0$ [9]. To reveal whether or not the statement is correct, we consider the expansion of the field $h_{\mu v}$ around the Vacuum Expectation Value $(\mathrm{VEV}) h_{\mu \nu}^{\mathrm{VEV}}$

$$
h_{\mu v}=h^{\mathrm{VEV}}+a_{\mu v}+i b_{\mu v}
$$

where $a_{\mu v}$ and $b_{\mu v}$ are real. Substituting the expression 6.10 into the potential term, we find the quadratic part taking the following form:

$$
\begin{aligned}
\mathscr{L}_{\mathrm{BP}}^{(2)}= & \frac{1}{2} \eta^{\mu_{1} v_{1} \mu_{2} v_{2} \mu_{3} v_{3}} \partial_{\mu_{1}} a_{\mu_{2} v_{2}} \partial_{v_{1}} a_{\mu_{3} v_{3}}+\frac{1}{2} m_{a}^{2} \eta^{\mu_{1} v_{1} \mu_{2} v_{2}} a_{\mu_{1} v_{1}} a_{\mu_{2} v_{2}} \\
& +\frac{1}{2} \eta^{\mu_{1} v_{1} \mu_{2} v_{2} \mu_{3} v_{3}} \partial_{\mu_{1}} b_{\mu_{2} v_{2}} \partial_{v_{1}} b_{\mu_{3} v_{3}} .
\end{aligned}
$$


This seems to mean that the theory contains one massive spin two mode and one massless spin two mode which corresponds to a Nambu-Goldstone mode. On the other hand, the nonderivative interactions are given as follows:

$$
\begin{aligned}
\mathscr{L}_{\text {interactions }}= & \sqrt{\frac{\lambda}{24}} m_{a} \eta^{\mu_{1} v_{1} \mu_{2} v_{2} \mu_{3} v_{3}} a_{\mu_{1} v_{1}} a_{\mu_{2} v_{2}} a_{\mu_{3} v_{3}}+\sqrt{\frac{\lambda}{24}} m_{a} \eta^{\mu_{1} v_{1} \mu_{2} v_{2} \mu_{3} v_{3}} a_{\mu_{1} v_{1}} b_{\mu_{2} v_{2}} b_{\mu_{3} v_{3}} \\
& +\frac{\lambda}{4 !} \eta^{\mu_{1} v_{1} \mu_{2} v_{2} \mu_{3} v_{3} \mu_{4} v_{4}} b_{\mu_{1} v_{1}} b_{\mu_{2} v_{2}} b_{\mu_{3} v_{3}} b_{\mu_{4} v_{4}}+\frac{\lambda}{4 !} \eta^{\mu_{1} v_{1} \mu_{2} v_{2} \mu_{3} v_{3} \mu_{4} v_{4}} a_{\mu_{1} v_{1}} a_{\mu_{2} v_{2}} a_{\mu_{3} v_{3}} a_{\mu_{4} v_{4}} \\
& +\frac{\lambda}{3 ! \cdot 2} \eta^{\mu_{1} v_{1} \mu_{2} v_{2} \mu_{3} v_{3} \mu_{4} v_{4}} a_{\mu_{1} v_{1}} a_{\mu_{2} v_{2}} b_{\mu_{3} v_{3}} b_{\mu_{4} v_{4}} .
\end{aligned}
$$

This shows that the nonlinear potential for $b_{\mu \nu}$ exists although the mass term for $b_{\mu \nu}$ is absence. Thus, in this case, the theory cannot be defined around nontrivial vacua.

\section{Summary}

We construct new theories of massive spin two fields and study properties of nontrivial vacua. We find that stable vacua always have higher energy than the unstable vacua. Furthermore, for the charged $U(1)$ fields, there is no stable, nontrivial vacua .

\section{References}

[1] C. de Rham and G. Gabadadze, Phys. Rev. D 82, 044020 (2010) [arXiv:1007.0443 [hep-th]]; C. de Rham, G. Gabadadze and A. J. Tolley, Phys. Rev. Lett. 106, 231101 (2011) [arXiv:1011.1232 [hep-th]].

[2] S. F. Hassan and R. A. Rosen, Phys. Rev. Lett. 108, 041101 (2012) [arXiv:1106.3344 [hep-th]].

[3] S. F. Hassan and R. A. Rosen, JHEP 1204 (2012) 123 [arXiv:1111.2070 [hep-th]].

[4] S. F. Hassan and R. A. Rosen, JHEP 1107 (2011) 009 [arXiv:1103.6055 [hep-th]].

[5] K. Hinterbichler, JHEP 1310 (2013) 102 [arXiv:1305.7227 [hep-th]].

[6] M. Fierz and W. Pauli, Proc. Roy. Soc. A 173, 211 (1939).

[7] D. G. Boulware and S. Deser, Phys. Rev. D 6, 3368 (1972);

D. G. Boulware and S. Deser, Annals Phys. 89, 193 (1975).

[8] Y. Ohara, S. Akagi and S. Nojiri, Phys. Rev. D 92, 023011 (2015) [arXiv:1407.5765 [hep-th]].

[9] Y. Ohara, Phys. Rev. D 94, 083004 (2016) [arXiv:1606.07879 [hep-th]]. 